\title{
El DISEÑO DE UN MODELO DE GESTIÓN DE LA RELACIÓN CON LOS CLIENTES PARA LAS MICRO Y PEQUEÑAS EMPRESAS
}

\author{
DESIGN OF A MODEL OF CUSTOMER RELATIONSHIP MANAGEMENT FOR MICRO \\ AND SMALL ENTERPRISES
}

\author{
${ }^{1}$ Ampelio Ferrando Perea
}

\begin{abstract}
Resumen
La presente investigación tuvo por objetivo conocer la situación de las micro y pequeñas empresas comerciales minoristas de venta de productos de consumo de la zona comercial del distrito de Jesús María de Lima Metropolitana en cuanto al desarrollo de actividades de búsqueda, captación y fidelización de clientes para diseñar un modelo integral de gestión de las relaciones con el cliente. Para lograr este objetivo la investigación se hizo mediante una encuesta a 100 micros y pequeñas empresas. El análisis de los resultados mostró que solo el 33\% dice conocer acerca de técnicas de gestión de la relación con el cliente, $20 \%$ que cuenta con alguna aplicación computarizada con la que gestiona sus clientes y que el $61 \%$ estarían dispuestas a implementar software para gestionar las relaciones con los clientes, razones por las que se concluye que es factible implementar un modelo de gestión de las relaciones con los clientes en las pequeñas empresas mencionadas.
\end{abstract}

Palabras claves: CRM, Marketing relacional, retención del cliente, software CRM, Modelo CRM.

\begin{abstract}
This research aimed to determine the status of micro and small commercial retail sales of consumer products from the shopping area of Jesus Maria district of Metropolitan Lima in terms of developing customer search, attracting and loyalty activities to design a comprehensive model management of customer relationships. To achieve this goal it was done through a survey of 100 micro and small enterprises. The analysis of the results showed that only $33 \%$ say they know about management techniques behind customer relationship, 20\% have a computer application with which you manage your clients and that $61 \%$ would be willing to implement software to manage customer relationships, reasons for concluding that it is feasible to implement a model of customer relationships management in small companies mentioned.
\end{abstract}

Key words: CRM, relationship marketing, customer retention, CRM Software, CRM model.

\section{Introducción}

Actualmente para las empresas, la relación con el cliente resulta una actividad primordial cualquiera que sea su giro. Ninguna empresa puede existir si no tiene clientes que atender con sus productos y servicios, por ello gran número de empresas dedican tiempo y esfuerzos para incrementar su cartera de clientes y la satisfacción que estos puedan lograr. Por consiguiente, conservarlos es para todas ellas un reto cotidiano que tienen que afrontar en el mundo competido y globalizado de hoy en día.

Las organizaciones tienden a enfocarse con gran fuerza en sus clientes utilizando la CRM ("Customer Relationship Management o gestión de relaciones con clientes"), modelo de administración que permite capturar y analizar la información proveniente de los clientes que facilite la toma de decisiones para la personalización de los productos que sirve para atraer, retener y profundizar las relaciones con los clientes según el nivel de rentabilidad de cada uno de ellos. Buljan (2011), lo define como: "una estrategia de negocio, apoyada por una estructura de procedimientos y tecnología, que mejora la adquisición, la retención y el crecimiento de mis clientes". (1). La clave de su uso está en retener los clientes más rentables, sin miedo a dejar que se alejen aquellos que ofrecen una baja rentabilidad.

La importancia de retención de los clientes para las utilidades de una empresa se basa en estudios que confirman que es mucho más caro ganar un nuevo cliente que mantener aquellos que ya los tiene. Kotler (2006) señala que la pérdida de clientes rentables repercute considerablemente en las utilidades de una empresa. El costo de captar nuevos clientes es cinco veces superior al costo de mantener satisfecho a un cliente actual. (2). Por consiguiente, al conformar las micro y pequeñas empresas la mayor parte del sistema productivo en nuestro país, se hace evidente la necesidad de implementar programas

\footnotetext{
${ }^{1}$ Universidad Nacional graria La Molina,Lima, Perú. E-mail: ampelio@lamolina.edu.pe
} 
de relación con el cliente para incrementar su número y desarrollar su fidelidad hacia los negocios.

Atraer nuevos clientes sigue siendo una tarea indiscutible, sin embargo las empresas deben concentrarse también en conservar los clientes actuales y forjar relaciones rentables y duraderas con ellos. Es por esto que se esta desarrollando una nueva forma de visualizar el marketing, una visión que implica crear, mantener y mejorar las relaciones sólidas con los clientes u otros interesados, un marketing relacionado al largo plazo, cuya meta es proporcionar valor a los clientes y a la medida el éxito de la empresa en el futuro.

El estudio tiene los siguientes objetivos:

- Conocer la situación de las micro y pequeñas empresas comerciales minoristas de venta de productos de consumo de la zona comercial del distrito de Jesús María de Lima Metropolitana en cuanto al desarrollo de actividades de búsqueda, captación y fidelización de clientes para diseñar un modelo integral de gestión de las relaciones con el cliente.

- Desarrollar el concepto "marketing relacional" y reconocer su importancia en el desarrollo y la perdurabilidad de las micro y pequeñas empresas comerciales minoristas.

- Proponer un modelo de gestión de las relaciones con el cliente que pueda ser aplicado por las micro y pequeñas empresas minoristas de venta de productos de consumo masivo.

\section{Materiales y métodos}

\section{Tipo de la investigación.}

La presente investigación ha seguido las pautas de tipo:

Descriptivo: describe los diversos hechos en la realidad problemática las mismas que se constituyeron en las causas incidentes que motivaron la necesidad de desarrollar la presente investigación, el fenómeno de estudio.

Observacional: los diversos fenómenos o acontecimientos han sido observados de tal manera que permiten un análisis e interpretación real de los hechos.

\section{Diseño de la investigación.}

La presente investigación es de diseño no experimental seccional transversal pues se investigó la situación de la gestión de la relación con el cliente de las mypes comercializadoras del damero comercial del distrito de Jesús María de Lima Metropolitana en un momento determinado de tiempo.

\section{Población y muestra.}

Se encuestó a 100 pequeñas empresas comercializadoras de bienes y servicios del Damero Comercial del distrito de Jesús María de Lima Metropolitana.

\section{Limitaciones del estudio.}

Este trabajo de investigación presenta varias limitaciones:

a) La primera limitación es el lugar, se realizó únicamente en el Damero Comercial del Distrito de Jesús María.

b)La segunda limitación fue el tipo de participantes. Los micros y pequeños empresarios, en algunos casos, mostraron reserva para brindar información y permitir la observación de sus locales, condicionando la realización de la encuesta a aquellos establecimientos comerciales en los cuales sus propietarios o administradores han estado dispuestos a colaborar con la realización del estudio.

c) También ha constituido una limitación importante la disponibilidad de recursos financieros para la investigación constituido por los fondos asignados del FEDU.

\section{Resultados y discusión}

En esta sección se presentan los resultados del estudio realizado a través de encuesta sobre gestión de la relación con los clientes aplicada a los responsables de las tiendas del Damero comercial del Distrito de Jesús María de Lima metropolitana.

\section{Factor fundamental de subsistencia de la empresa.}

Los resultados de la encuesta ponen de manifiesto que el $87 \%$ de los responsables de las mypes comerciales de Jesús María consideran que el cliente es fundamental para la empresa, frente a un $8 \%$ que considera que lo fundamental son los precios bajos, un $4 \%$ la competencia y un $1 \%$ los trabajadores. Ver Tabla 01 siguiente.

Tabla 1. Factor fundamental de subsistencia de la empresa.

\begin{tabular}{lcc}
\hline \multirow{2}{*}{ Pregunta } & \multicolumn{2}{c}{ Resultado } \\
\cline { 2 - 3 } & $\mathrm{Q}$ & $\%$ \\
\hline ¿Conoce usted técnicas de & & \\
gestión de las relaciones con & 100 & 100 \\
los clientes o CRM? & & \\
- Sí & 33 & 33 \\
- No & 67 & 67 \\
\hline
\end{tabular}

Fuente: Elaboración propia

Los resultados mencionados anteriormente son coincidentes con lo planteado por Emprendedores (2011): "El activo "cliente" es clave para desarrollar una estrategia de rentabilidad de una empresa. Gran parte de las posibilidades de crecimiento de una empresa pasan por gestionar de la forma más rentable posible sus activos, y muy especialmente el activo "cliente". Incluso cuando el crecimiento "en cantidad" de consumidores es factible, la mejora en el aporte de cada uno es una estrategia que irá en directa relación con ganar en "calidad" de clientes."(3). El enfocarse en clientes que sean rentables es un objetivo en que vale la pena fijarse. La eficiencia es una forma de ahorrar recursos humanos y financieros, además de proveer a una organización de los clientes adecuados a quienes ofrecer productos adecuados para ellos (4). 


\section{Importancia del cliente para la empresa.}

En la tabla 02 puede verse que el $87 \%$ de los responsables de las mypes encuestadas consideran al cliente como muy importante para la empresa, y un $13 \%$ como importante.

Tabla 2. Importancia del cliente para la empresa.

\begin{tabular}{lcc}
\hline \multirow{2}{*}{ Pregunta } & \multicolumn{2}{c}{ Resultado } \\
\cline { 2 - 3 } & $\mathrm{Q}$ & $\%$ \\
\hline ¿Qué tan importante cree Ud. & 100 & 100 \\
que es el cliente en una empresa? & 87 & 87 \\
- Muy importante & 13 & 13 \\
- Importante & 0 & 0 \\
- No tan importante & 0 & 0 \\
- Nada importante &
\end{tabular}

Tales resultados se deben principalmente a que: los productos y servicios son cada día más parecidos, los clientes más valiosos son conscientes de su valor y, por lo tanto mucho más exigentes y menos fieles que antes. Los competidores siempre pueden copiar o comercializar el mismo producto, o venderlo más barato; pero la forma de mantener relaciones exitosas con los clientes es difícil de reproducir. Por tal razón, los comportamientos, actitudes y valores creados para satisfacer las necesidades de los clientes significan la verdadera ventaja competitiva; es necesario identificar que es lo que los clientes piensan y que esperan alcanzar ya que esta será la mejor forma de saber el tipo de futuro que le espera a la empresa.(5).

\section{Conocimiento de técnicas de gestión de la relación con los clientes}

Los resultados de la encuesta en la Tabla 03 siguiente señalan que el $67 \%$ de los responsables no conocen las técnicas de gestión de las relaciones con los clientes, frente a un $33 \%$ que dicen conocerlas.

Tabla 3. Conocimiento de técnicas de gestión de la relación con los clientes.

\begin{tabular}{lcc}
\hline \multirow{2}{*}{ Pregunta } & \multicolumn{2}{c}{ Resultado } \\
\cline { 2 - 3 } & $\mathrm{Q}$ & $\%$ \\
\hline $\begin{array}{l}\text { ¿Qué o quién es fundamental para } \\
\text { la subsistencia de una empresa } \\
\text { como la de usted? }\end{array}$ & 100 & 100 \\
- El cliente & 87 & 87 \\
- Los precios bajos & 8 & 8 \\
- La competencia & 4 & 4 \\
- Los trabajadores & 1 & 1 \\
- Las leyes peruanas Mype & 0 & 0 \\
\hline Fuente: Elaboración propia & &
\end{tabular}

Visto tales resultados, aunque el conocimiento de las técnicas sobre Administración de las Relaciones con los Clientes es muy importante para las mypes encuestadas, las dos terceras partes de ellas las desconocen. Por consiguiente, es necesario que las conozcan aceptando el reto de lograr que en todos los puntos en que se interactúe con ellos, seas capaz de ofrecer un valor agregado, obteniendo con ello su satisfacción y fidelidad.

La evolución de los negocios ha hecho que se deje de "vender lo que el consumidor necesita". Ahora, el enfoque está precisamente en lograr una "administración rentable de las relaciones con el mercado". Esto se consigue cuando todo el personal de la empresa se centra en tres puntos principales:

- Búsqueda y captación de nueva clientela. La información es poder, y mientras mejor enterado estés acerca de tu mercado, mejores armas tendrás para conservarlo.

- Lograr su fidelidad. El CRM hace que todo el personal de tu empresa tenga contacto con los clientes y posea información de ellos. Esto se traduce en un excelente servicio que se verá reflejado en el índice de satisfacción que clientes actuales y futuros tendrán tras haber hecho negocios contigo.

- Orientarse hacia mantener la rentabilidad con ellos en el largo plazo.(6)

\section{Factores importantes para que un cliente vuelva a realizar una nueva compra.}

Los factores más importantes considerados por los conductores de las mypes comerciales de Jesús María figuran: Buena atención con $86 \%$, calidad de productos y servicios con $69 \%$, precios accesible $65 \%$, Promociones, $20 \%$ y surtido $10 \%$. Otros factores figuran con porcentajes inferiores al 10\%. Ver Tabla 04 siguiente.

Tabla 4. Factores importantes para que un cliente vuelva a realizar una nueva compra.

\begin{tabular}{lcc}
\hline \multirow{2}{*}{ Pregunta } & \multicolumn{2}{c}{ Resultado } \\
\cline { 2 - 3 } & Q & $\%$ \\
\hline ¿Qué factores considera importantes en & & \\
su tienda para que un cliente vuelva a & 100 & 100 \\
realizar una nueva compra? & 86 & 86 \\
Buena atención & 69 & 69 \\
Calida & 65 & 65 \\
Precio accesible & 20 & 20 \\
Promociones & 10 & 10 \\
Surtido & 8 & 8 \\
Buen ambiente en local & 8 & 8 \\
Exposición de productos & 7 & 7 \\
Descuentos & 4 & 4 \\
Cercanía al local & 4 & 4 \\
Horarios & 2 & 2 \\
Exclusividad & 2 & 2 \\
Garantías & 2 & 2 \\
Publicidad & 2 & 2 \\
Entrega a domicilio & 1 & 1 \\
Marca & 1 & 1 \\
Obsequios & &
\end{tabular}

Los resultados en la tabla 04 denotan que, a través de los factores analizados, se observa que, hay empresas pequeñas en las que la CRM se ha integrado sin mayor 
esfuerzo; lo único que hacen es estar en contacto permanente con un pequeño número de personas e inventar nuevas formas de dar una buena impresión a sus clientes, comercializar para ellos, venderles productos y darles servicio. Es por ello, que Swift (2002) dice: "la historia del servicio y de atención al cliente, así como la administración de la relación con este, no es sino un intento de recuperar la intimidad de que gozaban los pequeños comerciantes de antaño, gracias a que la clientela vivía cerca de ellos; clientes y vendedores estaban unidos no solo por vínculos comerciales, sino también por lazos familiares y comunitarios" (7). De esta manera, las actividades de marketing y de gestión de los clientes estarán centradas en la búsqueda de una nueva fuente de ventajas competitivas basadas en la diferenciación e implicación emocional de los clientes y en la creación de experiencias positivas ligadas a la marca y/o establecimiento.

\section{Escucha de la voz del cliente.}

Los encuestados manifiestan que atienden lo que dicen sus clientes mediante: Atención de quejas en un $45 \%$, encuestas de satisfacción en un $23 \%$, entrevista a clientes $14 \%$, grupo focal $2 \%$. No respondieron a la pregunta $16 \%$. Ver Tabla 05 siguiente.

Tabla 5. Escucha de la voz del cliente.

\begin{tabular}{lcc}
\hline \multirow{2}{*}{ Pregunta } & \multicolumn{2}{c}{ Resultado } \\
\cline { 2 - 3 } & Q & $\%$ \\
\hline Para orientar la empresa al cliente es & & \\
necesario escuchar la voz del cliente. iEn & 100 & 100 \\
el caso de su empresa, como se realiza? & & \\
Solo atiende quejas & 45 & 45 \\
Encuesta de satisfacción & 23 & 23 \\
Entrevista a clientes & 14 & 14 \\
Grupo focal & 2 & 2 \\
No sabe, no contesta & 16 & 16 \\
\hline
\end{tabular}

Fuente: Elaboración propia.

La preferencia de los encuestados por la atención de quejas es coincidente con lo manifestado por la CEEI (2011): "a fin de convertir una situación negativa con un cliente en una situación positiva, es importante que la empresa y todo el personal que pueda estar en contacto con el cliente, sepa cómo tratar las quejas y reclamaciones con eficacia para conseguir recuperar el servicio y la satisfacción del cliente, de modo que las reciban con una actitud y un comportamiento correctos... Para conseguir la satisfacción de los clientes y por tanto su fidelidad, la respuesta definitiva debe ser satisfactoria y debe ser comunicada a la mayor brevedad. (8). El análisis de la voz del cliente puede ayudar a la empresa a hacer entender a toda la organización cuál es la dirección correcta para alinearse con las expectativas y necesidades del cliente.

\section{Uso de aplicación computarizada con la que gestionan clientes}

En relación a este factor, en la tabla 06 se aprecia que, los encuestados manifiestan que atienden lo que dicen sus clientes a través de: Atención de quejas en un $45 \%$, encuestas de satisfacción en un $23 \%$, entrevista a clientes $14 \%$, grupo focal $2 \%$. No respondieron a la pregunta $16 \%$. Aquellos encuestados que contestaron positivamente expresaron que las aplicaciones que utilizan son: hoja de cálculo Excel 9\%, correo electrónico 9\% y facebook 2\%.

Tabla 6. Uso de aplicación computarizada con la que gestionan clientes.

\begin{tabular}{llcc}
\hline \multicolumn{2}{c}{ Pregunta } & \multicolumn{2}{c}{ Resultado } \\
\cline { 3 - 4 } & & $\mathrm{Q}$ & $\%$ \\
\hline ¿La empresa cuenta con alguna & & \\
aplicación computarizada con la que & 100 & 100 \\
gestione sus clientes? & & \\
- Sí & 20 & 20 \\
- No & 71 & 71 \\
- No sabe, no contesta & 9 & 9 \\
\hline
\end{tabular}

Fuente: Elaboración propia

Estos resultados denotan, que muchas empresas confunden el CRM con un programa de software y no entienden que primero hay que diseñar una estrategia y desarrollarla internamente. Cabe señalar al respecto que siempre hay que tener en cuenta que el modelo CRM hace tanto referencia a la estrategia de negocio focalizada hacia el cliente, como a toda las aplicaciones informáticas, tanto software como hardware conocidas como front office, necesarias para procesar, analizar y exponer la información resultante para medir y retroalimentar la estrategia de negocio desarrollada.(90). El software es la herramienta de apoyo para el desarrollo de la estrategia CRM.

\section{Implementación de nuevo software para gestionar las relaciones con el cliente.}

En la tabla 07 a continuación se aprecia que los encuestados manifiestan en un $64 \%$ que si están dispuestos a implementar nuevo software para gestionar la relación con sus clientes frente a un 9\% que dijeron que no. El 27\% considero que necesitaría analizar la posibilidad de su uso.

Tabla 7. Implementación de nuevo software para gestionar las relaciones con el cliente.

\begin{tabular}{lccc}
\hline \multirow{2}{*}{ Pregunta } & \multicolumn{2}{c}{ Resultado } \\
\cline { 2 - 4 } & $\mathrm{Q}$ & $\%$ \\
\hline ¿Si hubiera otra herramienta tecnológica \\
(software) que permita gestionar las \\
relaciones con sus clientes, usted la & & \\
implementaría en su empresa? & & 100 \\
- Sí & 64 & 64 \\
- No & 9 & 9 \\
- La analizaría & 27 & 27 \\
\hline Fuente: Elaboración propia. & &
\end{tabular}


El 9\% de los responsables de las mypes comerciales que respondieron no, aducieron que era debido a: costo $6 \%$, no tienen personal capacitado para su uso $1 \%$, no disponen de tiempo para implementarlo $1 \%$ y no es necesario $1 \%$.

Al respecto, Hernández (2007) señala que los principales motivadores que llevan a las empresas a instalar una tecnología de este tipo son:

- Incrementar el nivel de satisfacción de los clientes.

- Retener los clientes actuales.

- Incrementar el tiempo de vida de un cliente.

- Proveer información estratégica a la empresa.

- Atraer nuevos clientes.

- Ahorro en costos. (10)

\section{Propuesta de un modelo de gestión de las relaciones} con los clientes.

A la hora de diseñar un programa de fidelización de clientes no interesa que todos los clientes participen, sino que esté orientado hacia su fuente de energía, sus mejores clientes, los que aportan márgenes saludables y tienen el favor potencial futuro para la empresa. Estos son los clientes que se necesita conocer mejor, para en un futuro poder predecir sus necesidades y de esta manera poder diseñar productos y servicios que se ajusten mejora sus necesidades y así poderlos vender mejor.

Por consiguiente, el modelo presenta las siguientes características:

- Es orientado hacia el cliente basado en el marketing relacional.

- Es una estrategia de negocio que implica: elaboración de planes operativos y de acción a mediano y largo plazo y la alineación de la organización empresarial.

- Tiene por finalidad generar impactos directos sobre: la fidelización de los clientes (retención), rotación de ventas (Upper-selling y cross-selling), captación de nuevo negocio a través de la detección de nuevas oportunidades y reducción del costo de venta.

Asimismo, el modelo CRM propuesto se basa en tres elementos fundamentales: una buena estrategia de negocio, apoyada por una herramienta tecnológica que tiene que ser correctamente utilizada por las personas, tanto administrativa como conceptualmente.

Estrategia: CRM es una estrategia de negocio por lo que será preciso orientar toda la empresa en torno a ella.

La estrategia válida para una solución CRM es una orientación verdadera y real al cliente, real porque no se trata sólo de una buena "Atención al cliente", sino que el cliente sea el centro de la empresa. Las decisiones se toman en torno a lo que el cliente espera y esto influirá directamente en la rentabilidad positiva de la empresa, porque permite conocerle mejor y construir a partir de sus intereses. Establecemos una estrategia diferenciada según tipología de cliente:
- Definición de tipologías:

- Segmentación. Un mismo segmento de clientes tiene intereses comunes que pueden responderse con una oferta similar.

- Priorización, establecer los clientes de valor, diferenciar entre clientes “A, B, C", "platino, oro, plata,...".

- Estrategias diferenciadas según tipología o segmento:

- Definir la relación a partir de la tipología del cliente.

○ Definir una propuesta de valor.

Herramienta Tecnológica: que debe estar al servicio de la estrategia.

Las empresas han recurrido a la filosofía de Manejo de Relaciones con el Cliente- CRM, que hoy no sólo está disponible en forma de software, también sobre Cloud Computing, una tendencia que cada día más Pymes están adoptando para reducir costos.

El CRM está disponible bajo demanda, es amigable y conforme la Pyme va creciendo, se van usando cada una de sus partes. Una vez que llega el momento de cambiar de modelo, la plataforma es compatible y flexible como para mover los componentes, incluso si la empresa creciera y se decidiera crear un datacenter, se pueden cambiar hacia la infraestructura del negocio y mantener otras aplicaciones en nube. (11)

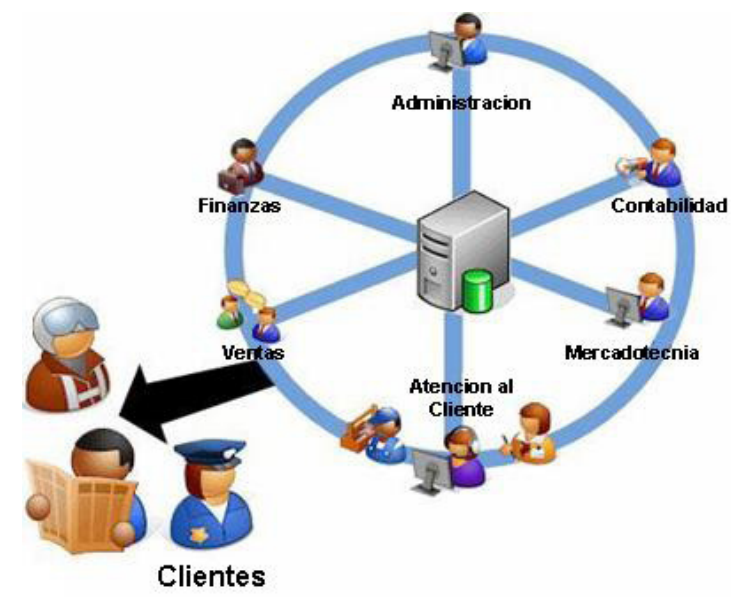

Figura 1. Modelo CRM para Pymes.

Fuente: Barrera G. 2011. CRM en la nube, una costeable opción Pyme. p.1

Algunos ejemplos, entre otros, de los softwares más recomendados son:

Salesforce: Este software para CRM es en definitiva merecedor de toda alabanza que ha recibido de varios usuarios y empresas. Es recomendable Salesforce para pequeña y grandes empresas con necesidad de software para CRM. Salesforce tiene las herramientas para que te hagas más eficiente y para fortalecer tu relación con los clientes, incrementar las ventas y los ingresos. 
Oncontact: Su diseño se centra en menús desplegables los cuales proporcionan un sinnúmero de opciones en cada una de las categorías de funciones. Tiene el diseño y capacidades que los usuarios más desean en un sistema de gestión de relación con el cliente y lo recomendamos como una de las mejores opciones de software para CRM de la actualidad.

Sage ACT: Es una buenísima opción de software para CRM. Los usuarios apreciarán el diseño simple y limpio de esta aplicación. La mayoría de los usuarios rápidamente se sienten con confianza tras abrir la aplicación y puede de inmediato comenzar a utilizar sus funciones y herramientas. Mientras que la aplicación no provee las características únicas y las herramientas que se encuentran en las primeras dos posiciones, si brinda todas las características y herramientas generales en las que tanto confían los usuarios.

Prophet: Es una buena opción de software para CRM que está hecho a la manera de Outlook. Aunque es fácil de usar y tiene una amplia gama de funciones y herramientas. A medida que los usuarios se acostumbran a la configuración y a las herramientas, se sienten más a gusto y pueden aprovechar todo lo que la aplicación tiene para ofrecer.

Relenta: Esta opción de software para CRM tiene grandes beneficios y funciones que se encuentran disponibles a sus usuarios. Aunque Relenta no ofrece todas las opciones que otras aplicaciones de gestión de relaciones con los clientes ofrecen, sí brinda una enorme colección. Es recomendable este software para CRM por su facilidad de uso, la navegación clara y sencilla, además de las herramientas y funciones que ofrece.

WebAsyst : Si estás buscando un sistema de gestión de relación con el cliente, brinda una opción para compartir fotos, muy útil en muchas circunstancias. Enfoca sus características de CRM para negocio en compartir archivos y la gestión de la relación con el cliente. Es una fabulosa opción para pequeñas empresas que poseen y operan una tienda online.

Chaos Intellect: Esta aplicación tiene una interfaz limpia y es fácil de usar y navegar. Generalmente, los usuarios estarán complacidos con sus herramientas y funciones eficientes que les permitirán acceder, organizar y usar la información desde una ubicación conveniente. Es recomendable para pequeñas empresas como opción de software para CRM. (12).

Al respecto, Murphy (2009) señala que para reducir la incertidumbre las empresas que buscan un CRM se están fijando en dos parámetros para elegir proveedor:
- Viabilidad del proveedor a largo plazo

- Funcionabilidad de la solución.(13)

1. Personas: La implantación de un Modelo CRM conlleva un cambio de cultura. La participación de las personas es fundamental para realizar y cambio, por lo que se requiere formación en contenidos y habilidades: estrategia y manejo de la herramienta. El aspecto más necesario es el motivacional, analizar los rechazos que se presentan e intentar solucionarlos para generar una actitud positiva hacia el sistema.

\section{Beneficios del modelo de Gestión de las Relaciones con los Clientes.}

El modelo pretende lograr tres tipos de mejoras en las micro y pequeñas empresas comerciales:

- Mejora de la productividad, mediante:

- Reducción de costos de explotación: producción, distribución, almacenamiento y los costos de transacciones internas.

- Ajuste a la demanda efectiva.

- Reducción de los costos de interacción con el cliente.

- Mejora de la competitividad, mediante:

- Cambio de enfoque al cliente.

- Dedicar entre el $80 \%$ al $90 \%$ a los clientes más importantes o con potencial de rendimiento.

- Potenciar la fuerza de ventas.

- Mejora de la gestión de contactos y oportunidades, mediante:

- Acortar el ciclo de ventas.

- Mejora de los porcentajes de cierre de las ventas.

- Origen de contactos variados.

- Evaluación de rendimiento de marketing y eficacia de las campañas comerciales.

- Identificación de tendencias.

- Control de equipo de ventas.

Implementación del modelo de Gestión de las Relaciones con los Clientes.

Elementos importantes a considerar en la implantación del modelo en las micro y pequeñas empresas son:

Definición de objetivos: Es prioritario tener en claro lo que se pretende conseguir a medio y a largo plazo con la implantación de estas acciones y también identificar los clientes más rentables con los que se van a realizar estos planes. 
Análisis de la orientación al cliente en la empresa: Se ha de comprobar si anteriormente se ha realizado algún estudio de satisfacción y en caso afirmativo se debería volver a analizar cuestionario y resultados. Es importante conocer las actividades formativas relativas al cliente ejecutadas por la empresa, así como la existencia de algún plan de comunicación externo con el cliente y los canales que estamos usando en este plan.

Análisis de bases de datos y sistemas de soporte informático. Se deben examinar las bases de datos que contengan información sobre los clientes, desde la herramienta tecnológica de soporte hasta saber cómo se capturan los datos o que tipo de información está recogida en estas bases de datos.

Estudio de la competencia. Se debe estar al día con respecto a las acciones que están realizando los competidores, ya que tal vez éstos puedan aportar ideas sobre las acciones que mejor o peor funcionan.

Diseño del programa dinámico de fidelización. Este proceso comprende una serie de acciones que se especifican a continuación:

- Analizar el tipo de clientes que participarán en el programa definido.

- Determinar las especificaciones técnicas de la herramienta informática de apoyo al programa de fidelización.

- Establecer los componentes de este plan: empleados que van a participar y que van a estar en contacto con los clientes, el programa de acciones a incorporar, tipos de tarjetas, catálogos, cupones, premios que se utilizarán, etc.

- Seleccionar la mejor herramienta de medida de satisfacción de los clientes.

- Implantar los parámetros de éxito del programa de fidelización.

- Definir los sistemas informáticos de soporte en función de los recursos disponibles.

Inversión. Evidentemente, el presupuesto con se cuenta para el establecimiento de un programa de fidelización es primordial, ya que normalmente estos planes cuentan con una pequeña partida sobre el total de la facturación. Una vez analizados estos aspectos, hay que decidir si hay que invertir en nuevos equipos o en desarrollar nuevas capacidades. Para ello es necesario conocer los costos de explotación, gestión y mantenimiento del sistema

Implantación del programa de fidelización. Una vez alcanzado este punto, la implantación del modelo de fidelización que se haya decidido consta de diversas etapas:
- Gestionar el programa de fidelización, teniendo en cuenta los costes de explotación, gestión y mantenimiento del sistema.

- Definir el sistema de mantenimiento y actualización periódica de la base de datos.

- Planificar los tiempos de implantación del programa.

- Establecer un plan de comunicación para los clientes que participen.

- Gestionar el cambio y la formación de los empleados.

- Incorporar las acciones y premios vinculados al plan de fidelización.

- Concretar los elementos integrantes del programa de fidelización y su participación en el programa.

- Establecer los parámetros de éxito del programa de fidelización y los sistemas informáticos de soporte.

- Producir los componentes del programa de fidelización: tarjetas, folletos, catálogos, etc.

Monitorización del programa de fidelización. Tras la implantación del programa de fidelización es preciso monitorizar y ajustar el plan dinámico de fidelización, hasta alcanzar los objetivos pre-definidos.

\section{Conclusiones}

La situación de las micro y pequeñas empresas comerciales minoristas de venta de productos de consumo de la zona comercial del distrito de Jesús María de Lima Metropolitana sobre gestión de las relaciones con el cliente manifiesta que solo el $33 \%$ dice conocer acerca de técnicas de gestión de la relación con el cliente, $20 \%$ que cuenta con alguna aplicación computarizada con la que gestiona sus clientes y que el $61 \%$ estarían dispuestas a implementar software para gestionar las relaciones con los clientes.

El marketing relacional resulta importante para la perdurabilidad de las micro y pequeñas empresas de Jesús María puesto que busca crear, fortalecer y conservar las relaciones de corto, mediano y largo plazo de la empresa con sus clientes, con el fin de lograr un mayor número y calidad posible de transacciones, acudiendo a herramientas de marketing, comunicaciones y relaciones públicas.

El modelo propuesto de gestión de las relaciones con el cliente puede implementarse con relativa facilidad en las micro y pequeñas empresas de Jesús María pues éstas tienen generalmente pocos clientes a quienes conocen y reaccionan con gran flexibilidad a sus necesidades, gustos y preferencias; no requiere grandes inversiones y puede incrementar su rentabilidad haciéndolas sostenibles en el tiempo.

\section{Literatura citada}

Buljan, S. 2011. CRM un cambio cultural en la empresa. http://buljanandpartners.com/media/documentos/ CRM,\%20un\%20cambio\%20cultural\%20de\%201a \%20 empresa.pdf. 
Kotler, P. y Lane K. 2006 Dirección de marketing. Duodécima edición. México. Pearson Educación. p.168

Emprendedores. 2011. La importancia del activo "Cliente" para la rentabilidad de la empresa p.1 http:// www.blog-emprendedor.info/la-importancia-del-activocliente-para-la-rentabilidad-de-la-empresa/

Swift, R. 2002. CRM como mejorar las relaciones con los clientes. Mexico.Prentice Hall Pearson Educacion. p. 17.

Zapata, Y. 2006. Un nuevo enfoque de fidelización del cliente,CRM.

http://www.gestiopolis.com/recursos6/Docs/Mkt/crmfidelizacion-del-cliente.htm

Valero, A. 2011. CRM: la vitamina que tu empresa necesita para mantenerse con una clientela saludable. p. 1 .

http://www.ideasparapymes.com/contenidos/clientescrm-pymes-customer-relationship-management.html

Swift, R. 2002. CRM como mejorar las relaciones con los clientes. Mexico.Prentice Hall Pearson Educacion. p. 26.

CEEI, Comunidad Valenciana.2011. Manual 07: Analisis de satisfaccion del cliente Centros Europeos de Empresas Innovadoras p. 22,26.

Web and Macros. 2011. CRM - Customer Relationship Management. p.1

http://www.webandmacros.com/crm.htm

Hernández, R. 2007.CRM, o el precio de no conocer a tus clientes, parteI. p.1

http://www.gestiopolis.com/administracion-estrategia/ estrategia/crm-importancia-de-conocer-tus-clientes-2.htm Barrera, G. 2011. CRM en la nube, una costeable opcion Pyme p.1 http://www.soyentrepreneur.com/crm-en-lanube-una-costeable-opcion-para-pymes.html

Juancarlos.2011. Los diez mejores programas de software CRM para gestión de contactos contactos. Tekniqueando. p.1.

http://www.tecnikeando.com/software/110411-los-10mejores-programas-de-software-crm-para-gestion-decontactos.html

Murphy, E. 2009. Making Smart CRM Choices for 2009 p 1 http://www.crmbuyer.com/rsstory/65953.html 